\title{
Suppression of cell death by the secretory form of N-terminal ERC/mesothelin
}

\author{
TEGEXIBAIYIN WANG ${ }^{1}$, KAZUNORI KAJINO $^{1}$, MASAAKI ABE $^{1}$, KE TAN $^{1}$, MASUMI MARUO $^{1}$, \\ GUODONG SUN $^{1}$, YOSHIAKI HAGIWARA ${ }^{2}$, MASAHIRO MAEDA ${ }^{2}$ and OKIO HINO ${ }^{1}$ \\ ${ }^{1}$ Department of Pathology and Oncology, Juntendo University School of Medicine, 2-1-1 Hongo, Bunkyo-ku, \\ Tokyo 113-8421; ${ }^{2}$ Immuno-Biological Laboratories Co. Ltd., 5-1 Aramachi, Takasaki, Gunma 370-0831, Japan
}

Received March 30, 2010; Accepted May 11, 2010

DOI: 10.3892/ijmm_00000451

\begin{abstract}
ERC/mesothelin is highly expressed in malignant mesothelioma, pancreatic cancer, and ovarian cancer. It is cleaved to a $30 \mathrm{kDa}$ N-terminal secretory form (N-ERC) and a $40 \mathrm{kDa}$ C-terminal membranous form (C-ERC). Several functions have been reported for full-length ERC (full-ERC) and C-ERC/mesothelin, such as in cell adhesion and invasion, stimulation of cell proliferation, and the suppression of cell death. However, there have been no studies to date on the function of secretory N-ERC, despite the fact that it is abundantly secreted into the sera of mesothelioma patients. In this study, we investigated whether $\mathrm{N}$-ERC could function as a secretory factor to stimulate tumor progression. Full-, N, or C-ERC was overexpressed in the human hepatocellular carcinoma cell line Huh7 that lacks endogenous expression of ERC/mesothelin. Changes in the rates of cell proliferation and cell death were determined, and the state of signal transducers was examined using various endpoints: total cell counts, trypan blue exclusion rate, BrdU incorporation rate, TUNEL assay, and the phosphorylation of ERK1/2 and Stat3. In cells overexpressing N-ERC, phosphorylation of ERK1/2 was enhanced and the rate of cell death decreased, leading to the increase of cell number. The culture medium containing the secretory N-ERC also had the activity to increase the number of cells. Our data suggested that one of the full-ERC functions reported previously was mediated by the secretory N-ERC.
\end{abstract}

\section{Introduction}

In earlier studies, we isolated the rat Erc (Expressed in renal carcinoma) gene that was abundantly expressed in renal carcinoma tissues of the Eker rat $(1,2)$. Mesothelin $(M S L N)$,

Correspondence to: Dr Okio Hino, Department of Pathology and Oncology, Juntendo University School of Medicine, 2-1-1, Hongo, Bunkyo-ku, Tokyo 113-8421, Japan

E-mail: ohino@juntendo.ac.jp

Key words: ERC/mesothelin, secretory form, cell death, MAP kinase the human homologue of rat Erc, was identified as the antigenic target of a monoclonal antibody that reacted with a cell surface protein of malignant mesothelioma (MM) and ovarian cancer (3). ERC/mesothelin is expressed in normal mesothelium, and shows enhanced expression in malignancies such as MM, ovarian cancer, and pancreatic cancer (4-8). The signal peptide adjacent to the $\mathrm{NH}_{2}$-terminus, the centrally located furin-protease recognition site, and the glycosylphosphatidylinositol (GPI) anchorage sequence adjacent to the $\mathrm{COOH}$ terminus are conserved in both rat and human proteins. The ERC/mesothelin gene encodes a $70 \mathrm{kDa}$ precursor protein that is cleaved to an $\mathrm{NH}_{2}$-terminal $30 \mathrm{kDa}$ protein (N-ERC) and a $\mathrm{COOH}$-terminal $40 \mathrm{kDa}$ protein (C-ERC). N-ERC, which was also isolated as megakaryocyte potentiating factor (MPF) (9), is secreted into the extracellular space. C-ERC is attached to the cellular membrane via a GPI anchor, although some C-ERC molecules are also shed into the extracellular space.

Recently, an ELISA system for detecting N-ERC in human serum was established, and use of this system showed that the concentration of secretory N-ERC is increased in the serum of MM patients $(10,11)$. We also reported that serum N-ERC is increased in Eker rats with renal cancer (12). With regard to the function of full-ERC/mesothelin, several studies have reported that ERC/mesothelin can enhance cell adhesion and invasion $(3,13,14)$, and can either stimulate cell proliferation (15) or suppress cell death $(16,17)$. However, there are no reports on the function of secretory $\mathrm{N}-\mathrm{ERC} /$ mesothelin although it is abundantly secreted into the sera of MM patients and of Eker rats with renal cancer. In the present study, we investigated the biological function of $\mathrm{N}-\mathrm{ERC}$, and found that it could stimulate the phosphorylation of the MAP kinase, ERK1/2; additionally, N-ERC could suppress cell death and lead to an increase in cell number. This range of functions is similar to those reported for full-ERC/mesothelin (16).

\section{Materials and methods}

Construction of full-, $N$ - and $C$-ERC expression plasmids. The pcDNA3.1(+) vector containing the full coding region of human ERC/mesothelin cDNA, pcDNA3.1(+) full-ERC (Full in Fig. 1A), was double digested with the restriction enzymes $S a c I I$ and $X b a I$ to remove the $\mathrm{C}$-ERC coding region. The 
remaining 6.4-kb fragment was blunted by Klenow and selfligated to produce the N-ERC expressing vector, pcDNA3.1(+) $\mathrm{N}-\mathrm{ERC}$ ( $\mathrm{N}$ in Fig. 1A). Similarly, a C-ERC expressing vector, pcDNA3.1(+) C-ERC (C in Fig. 1), was constructed by replacing the $0.8 \mathrm{~kb}$ XhoI-SacII fragment of pcDNA3.1(+) full-ERC with the synthesized linker shown at the bottom of Fig. 1A. The structures of pcDNA3.1(+) N- and C-ERC were verified by DNA sequencing.

Cell culture and transfection. The human hepatic cancer cell line Huh7, which lacks endogenous ERC/mesothelin expression, was maintained in DMEM (Sigma-Aldrich, St. Louis, MO) supplemented with $10 \%$ fetal bovine serum at $37^{\circ} \mathrm{C}$ in a $95 \%$ air $/ 5 \% \mathrm{CO}_{2}$ atmosphere. Twelve hours after seeding $5 \times 10^{5} \mathrm{Huh} 7$ cells on a $10-\mathrm{cm}$ plate, the cell cultures were transfected with $6 \mu \mathrm{g}$ of plasmid DNA (Fig. 1) and $18 \mu \mathrm{l}$ of FuGENE 6 (Roche Applied Science, Mannheim, Germany). pcDNA3.1(+) without an insert was used as the mock vector. After transfection, the cells were transferred to low adhesion surface plates (EZ-BindShut Microplate, Iwaki, Tokyo), or to glass bottom dishes (D11030, Matsunami, Osaka), and the serum concentration was reduced to $2 \%$ (low serum condition). The low adhesion surface plates served as the anchorageindependent condition, and the glass bottom dishes were used for the immunofluorescent staining.

Western blotting. Mouse monoclonal anti-human N-ERC (7E7) and anti-human C-ERC (22A31) antibodies were described previously $(11,18)$. Rabbit polyclonal anti-p44/42 MAPK (ERK1/2), rabbit monoclonal anti-phospho ERK1/2 (Thr202/Tyr204), rabbit polyclonal anti-Stat3, and rabbit monoclonal anti-phospho Stat3 (Tyr705) (\#9102, \#4376, \#9132, and \#9145, respectively) were purchased from Cell Signaling Technology Japan, and rabbit polyclonal anticyclin E (M20)(sc-481) from Santa Cruz Biotechnology, CA. Forty-eight hours after transfection, Huh7 cells and culture medium were harvested. The cellular lysate $(60 \mu \mathrm{g})$ and culture medium $(30 \mu \mathrm{l})$ were adjusted to be in a solution containing $2 \%$ SDS, $10 \%$ glycerol, $50 \mathrm{mM}$ Tris- $\mathrm{HCl}$ (pH 6.8) and $100 \mathrm{mM}$ dithiothreitol (DTT). After boiling for $2 \mathrm{~min}$, the samples were electrophoresed on $10 \%$ Laemmli gels and transferred onto nitrocellulose membranes (Protran BA85, Whatman GmbH, Dassel, Germany).

Proteins on the membranes were blocked in $1 \%$ skim milk in phosphate-buffered saline with $0.1 \%$ Tween-20 (PBS-T) for $1 \mathrm{~h}$ at room temperature (rt). The membranes were then incubated with either $1 \mu \mathrm{g} / \mathrm{ml}$ anti $\mathrm{N}-\mathrm{ERC} /$ mesothelin (7E7), $2 \mu \mathrm{g} / \mathrm{ml}$ anti C-ERC/mesothelin (22A31) at rt for $1 \mathrm{~h}$, or antip44/42 (ERK1/2) (1:1000 dilution), anti-phospho-p44/42 (ERK1/2) (Thr202/Tyr204) (1:100 dilution), anti-Stat3 (1:1000 dilution), anti-phospho Stat3 (Tyr705) (1:100 dilution) or anti-cyclin $\mathrm{E}$ (1:100 dilution) at $4^{\circ} \mathrm{C}$ for overnight in PBS-T with $1 \%$ skim milk. Goat anti-mouse or anti-rabbit Ig conjugated with peroxidase labeled-dextran polymer (Envision K4000 or K4002, respectively) (Dako, Glostrup, Denmark) was used as the secondary antibody at a dilution of 1:100 in $1 \%$ skim milk in PBS-T at $\mathrm{rt}$ for $1 \mathrm{~h}$. The ECL detection system (GE Healthcare, Buckinghamshire, UK) was employed to visualize ERC/mesothelin, ERK1/2, Stat3 and cyclin E on membranes.
$\mathbf{A}$

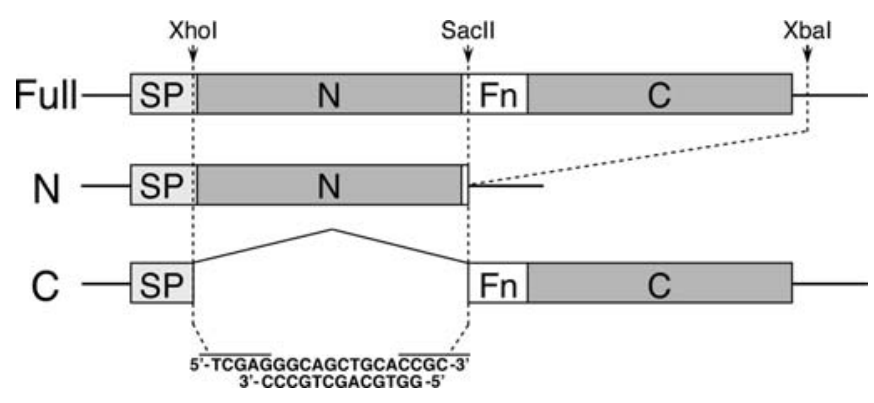

B

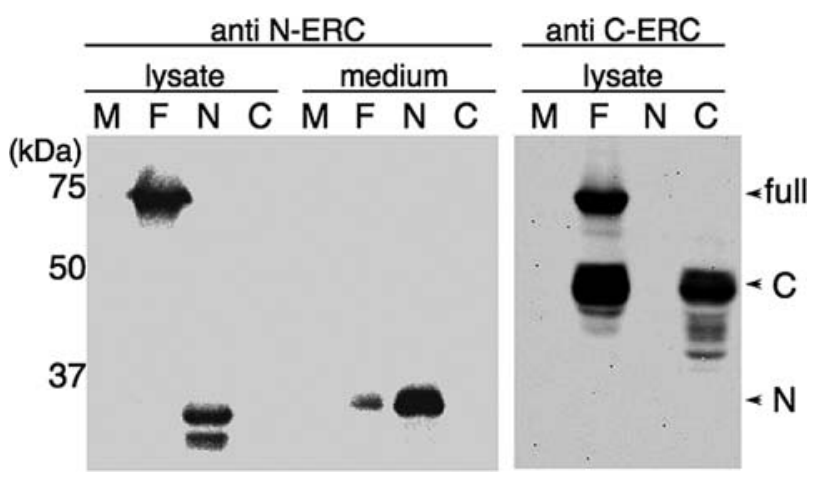

Figure 1. Full-, N-, C-ERC expression vectors and their overexpression. (A) Structures of pcDNA3.1(+)-full-ERC, -N-ERC, and -C-ERC are shown as Full, N, and C, respectively. XhoI, SacII and XbaI sites were used to construct the $\mathrm{N}$ - and C-ERC expression vectors from the Full-ERC sequence. The linker sequence used to replace the XhoI-SacII fragment of the Full-ERC in the construction of C-ERC is shown at the bottom of the image. SP, signal peptide; Fn, furin protease cleavage site. (B) Western blotting to confirm vector expression. Sixty micrograms of cellular lysate (lysate), or $30 \mu 1$ of culture medium (medium) from the Huh7 cultures were sampled $48 \mathrm{~h}$ after transfection and loaded onto $10 \%$ Laemmli gels. M, F, N, and C: mock, Full-, N-, and C-ERC, respectively.

Cell counting and cell viability assay in anchorageindependent cell cultures. After $12 \mathrm{~h}$ of transfection, $2 \times 10^{5}$ cells were transferred to $3-\mathrm{cm}$ low adhesion surface plates, and the serum concentration was reduced to $2 \%$. The number of total cells (Fig. 2A) and those showing trypan blue exclusion (Fig. 3A) were counted at 24, 48, and $72 \mathrm{~h}$ after passage.

BrdU (5-Bromo-2'-deoxy-uridine) incorporation assay. We used the BrdU labeling and detection kit I (11 296736001 , Roche Applied Science) with some modification of the manufacturer's recommendation. Briefly, $12 \mathrm{~h}$ after transfection, $1 \times 10^{5}$ cells were transferred to $3-\mathrm{cm}$ glass bottom dishes (D11030, Matsunami), and the serum concentration was reduced to $2 \%$. Forty-seven hours after passage, BrdU (final concentration $10 \mu \mathrm{M}$ ) was added to the medium and, $60 \mathrm{~min}$ later, the cells were fixed with $70 \%$ ethanol plus $15 \mathrm{mM}$ glycine ( $\mathrm{pH}$ 2.0). The fixed cells were incubated with a mouse monoclonal anti-BrdU mix containing nuclease, and then with an anti-mouse Ig-fluorescein. The cells were counterstained with 4'6-diamidino-2-phenylindole (DAPI) (Dojindo, Kumamoto, Japan). The frequency (\%) of BrdU positive cells per 1,000 DAPI (+) cells was determined in three different fields. Two replicate experiments were performed (Fig. 2B). 
A

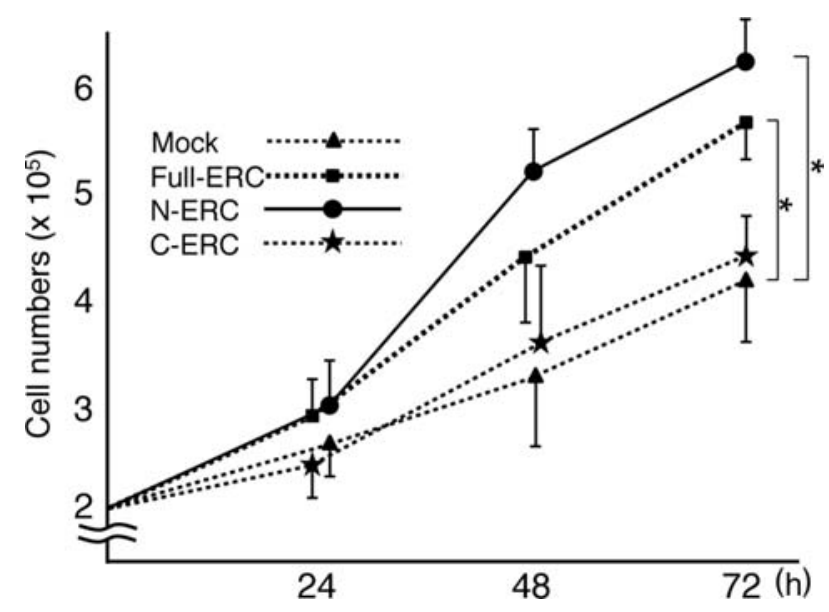

B

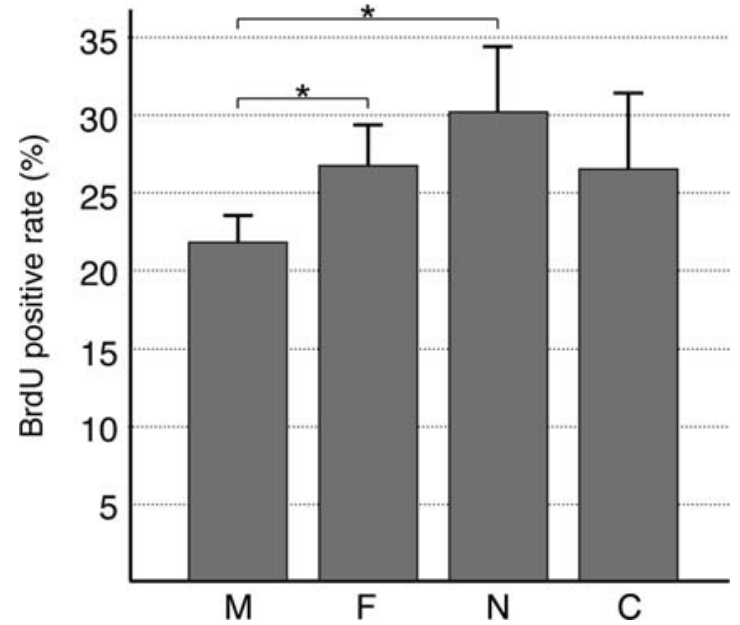

Figure 2. Effects of the expression of Full, N-, C-ERC on cell growth. (A) Total cell numbers under anchorage-independent conditions. Twelve hours after transfection, $2 \times 10^{5}$ cells were transferred to low adhesion surface plates ( $2 \%$ serum), and 24,48 , and 72 h later, total cell numbers were counted $(n=6) .{ }^{*} p<0.01$. (B) BrdU incorporation rate. Twelve hours after transfection, $1 \times 10^{5}$ cells were transferred to glass bottom dishes. Fortyseven hours after passage, BrdU $(10 \mu \mathrm{g})$ was added and, $1 \mathrm{~h}$ later, the cells were fixed with acidic ethanol. The frequencies (\%) of BrdU-positive cells in the populations of DAPI stained cells $(n=6)$ are shown. ${ }^{*} \mathrm{p}<0.01 . \mathrm{M}, \mathrm{F}, \mathrm{N}$, and $\mathrm{C}$ : as in Fig. 1B.

TUNEL (terminal deoxynucleotidyl transferase mediated dUTP nick-end labeling) assay. The DeadEnd Fluorometric TUNEL system (TB235, Promega, Madison, WI) was used with some modification of the manufacturer's recommendations. Briefly, $12 \mathrm{~h}$ after transfection, $1 \times 10^{5}$ cells were transferred to $3-\mathrm{cm}$ glass bottom dishes, and the serum concentration was reduced to $2 \%$. Seventy-two hours later, the cells were fixed for $25 \mathrm{~min}$ at $4^{\circ} \mathrm{C}$ with $4 \%$ methanol-free formaldehyde solution in PBS ( $\mathrm{pH} 7.4$ ), and permeabilized with $0.2 \%$ Triton $\mathrm{X}-100$ in PBS for 5 min. The cells were incubated at $37^{\circ} \mathrm{C}$ for 60 min with rTdT mix containing green fluorescein-12-dUTP, and counterstained with DAPI (Fig. 3B). The frequency (\%) of TUNEL positive cells per 1,000 DAPI $(+)$ cells was determined in three different fields. Two replicate experiments were performed (Fig. 3C).
Immunofluorescent staining of phosphorylated-ERK1/2. Twelve hours after transfection, $1 \times 10^{5}$ cells were transferred to $3-\mathrm{cm}$ glass bottom dishes, and the serum concentration was reduced to $2 \%$. Forty-eight hours later, the cells were fixed for $30 \mathrm{~min}$ at $4^{\circ} \mathrm{C}$ with $4 \%$ paraformaldehyde (Merck, Darmstadt, Germany) in PBS (pH 7.4). The cells were washed with PBS, incubated at $37^{\circ} \mathrm{C}$ for $1 \mathrm{~h}$ with rabbit monoclonal anti-phospho ERK1/2 (Thr202/Tyr204) (the same antibody as for Western blotting), and then with goat anti-mouse IgG conjugated Alexa Fluor 546 (A-11003, Molecular Probes, Invitrogen, Carlsbad, CA). The cells were counterstained with DAPI (Fig. 4B). The frequency (\%) of phospho-ERK1/2 positive cells per 1,000 DAPI (+) cells was determined in three different fields. Two replicate experiments were performed (Fig. 4C).

Culture of Huh7 cells in conditioned medium containing the secretory form of $\mathrm{N}$-ERC. After $24 \mathrm{~h}$ of transfection with mockor N-ERC-expressing vectors, culture medium was replaced with fresh one containing $2 \%$ FCS. Another 24 h later, the medium $(3 \mathrm{ml})$ was recovered and added to $3 \mathrm{~cm}$ low adhesion surface plates containing $2 \times 10^{5}$ non-treated Huh7 cells in 0.5 -ml medium. The numbers of cells were counted at 24,48 , and $72 \mathrm{~h}$ after the addition of conditioned medium (Fig. 5).

Statistical analysis. The data were analyzed using an F test in an analysis of variance, followed by Student's t-test (two-sided); $\mathrm{p}<0.01$ was regarded as statistically significant.

\section{Results}

Overexpression of full-, $N$-, and C-ERC/mesothelin. The basic structures of the three overexpressing plasmids pcDNA 3.1 (+)-full-ERC, -N-ERC, and -C-ERC are shown in Fig. 1A. By Western blotting, we confirmed expression of 70-kDa fulland 40-kDa C-ERC in cellular lysate, and a 30-kDa N-ERC in both lysate and culture medium. The N-ERC in the medium, derived from the N-ERC expressing vector, migrated to an identical position as that derived from the full-ERC expressing vector (Fig. 1B, left panel, lanes $\mathrm{F}$ and $\mathrm{N}$ in medium). Likewise, the C-ERC in the lysate, derived from the C-ERC expressing vector, was similar to that derived from the fullERC expressing vector (Fig. 1B, right panel, lanes $\mathrm{F}$ and $\mathrm{C}$ in lysate).

Promotion of cell growth by expression of full- or $\mathrm{N}$-ERC. The numbers of transfected Huh7 cells were counted at 24, 48 and $72 \mathrm{~h}$ after passage into anchorage-independent conditions (using low surface adhesion plates) and low (2\%) serum concentration (Fig. 2A). At $72 \mathrm{~h}$, full- and N-ERC transfected cells had significantly larger numbers of cells $(p<0.01)$ than mock or C-ERC transfected cells. To further analyze cell proliferative activity, we compared the amount of BrdU incorporated by the different cell groups after $1 \mathrm{~h}$ of labeling. Compared to the control (mock), full-, $\mathrm{N}$ - and C-ERC enhanced BrdU incorporation, but statistical significance was observed only for full- and N-ERC (Fig. 2B).

Effect of full- and N-ERC on cell viability. We conducted a trypan blue exclusion assay to estimate Huh7 cell viability at 
A

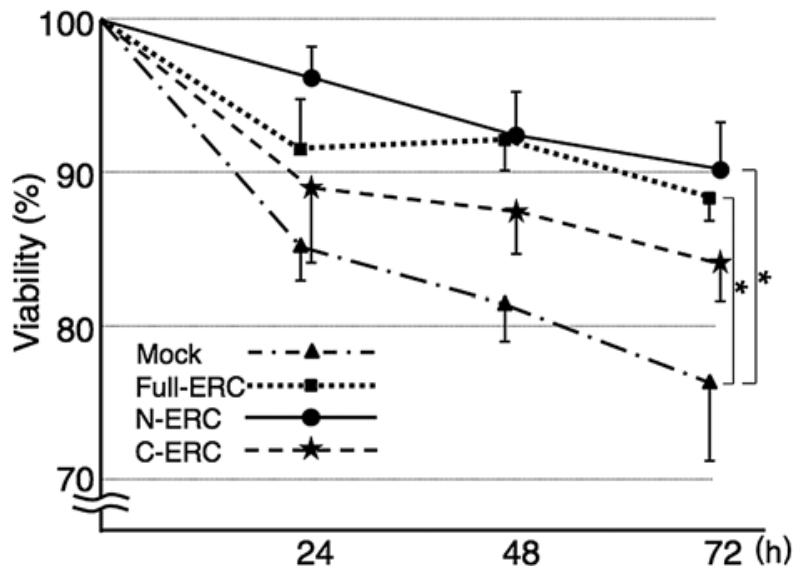

B
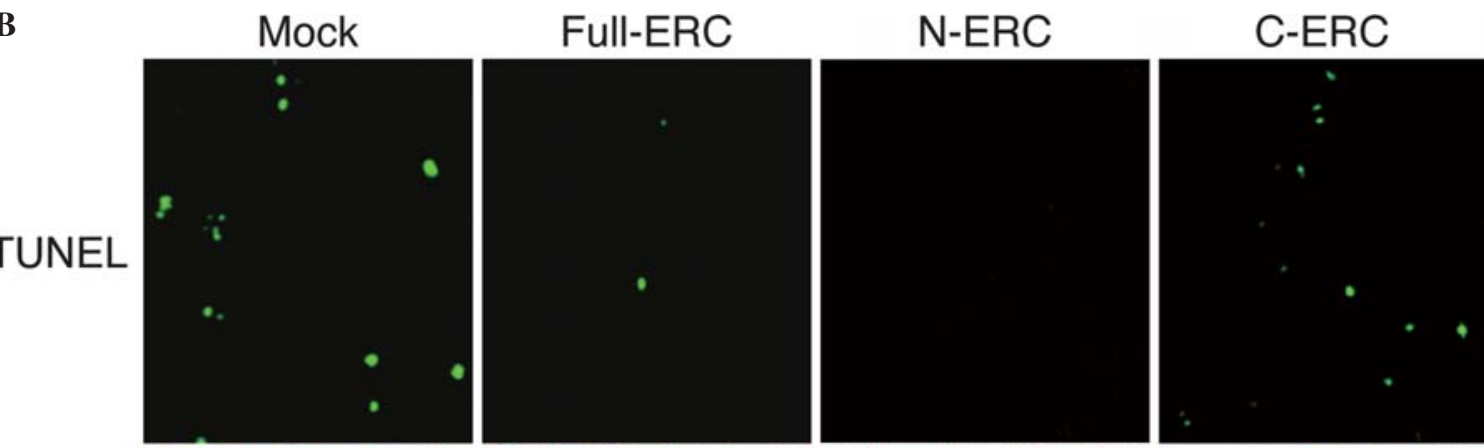

DAPI
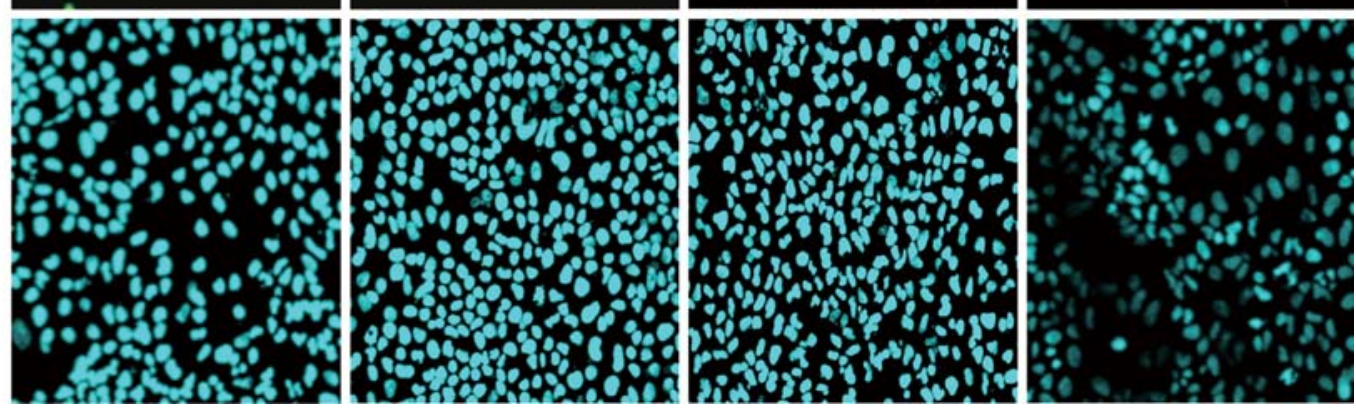

$\mathrm{C}$

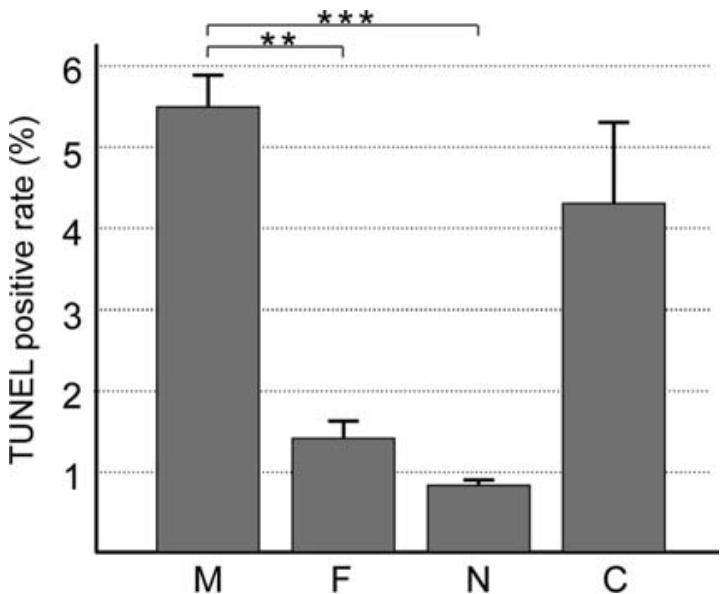

Figure 3. Effects of expression of full-, N-, or C-ERC on cell death. (A) Cell viability was determined by the proportions of cells showing trypan blue exclusion. Twelve hours after the transfection, $2 \times 10^{5}$ cells were transferred to the low adhesion surface plates ( $2 \%$ serum), and 24,48 , and $72 \mathrm{~h}$ later, $\%$ of trypan blue-excluding cells was counted. ${ }^{*} \mathrm{p}<0.01(\mathrm{n}=6)$. (B and C) TUNEL-positive cells were detected by immunofluorescent staining. Twelve hours after transfection, $1 \times 10^{5}$ cells were transferred to each glass bottom dish (2\% serum) and, $48 \mathrm{~h}$ later, the cells were fixed with $4 \%$ paraformaldehyde, and incubated with rTdT mix containing green fluorescein-12-dUTP, and counterstained with DAPI (B). The frequency (\%) of TUNEL-positive cells among the total population of DAPI-positive cells is shown $(\mathrm{C}) . \mathrm{M}, \mathrm{F}, \mathrm{N}$, and C: as in Fig. $1 \mathrm{~B} .{ }^{* *} \mathrm{p}=5.43 \mathrm{E}-10,{ }^{* * *} \mathrm{p}=5.22 \mathrm{E}-11$ $(\mathrm{n}=6)$.

24, 48 and $72 \mathrm{~h}$ after passage into anchorage-independent conditions and low serum concentration; cell viability was calculated as the proportion (\%) of trypan blue excluding cells in the total cell population (Fig. 3A). At $72 \mathrm{~h}$, full- and $\mathrm{N}$-ERC transfected cells showed significantly higher viability $(\mathrm{p}<0.01)$ than mock or C-ERC transfected cells. We carried out a TUNEL assay to examine cell death rates at the low serum concentration (Fig. 3B). Full- and N-ERC produced highly significant reductions in cell death rates compared to mock ( $\mathrm{p}=5.43 \mathrm{E}-10$ and 5.22E-11, respectively) (Fig. 3C).

ERK1/2 phosphorylation by full- and N-ERC. At $48 \mathrm{~h}$ after passage into anchorage-independent conditions (for the Western blot analysis) or into the glass bottom dishes (for immunofluorescent staining) and low serum concentration, ERC-transfected cells were harvested as described in Materials 
$\mathbf{A}$

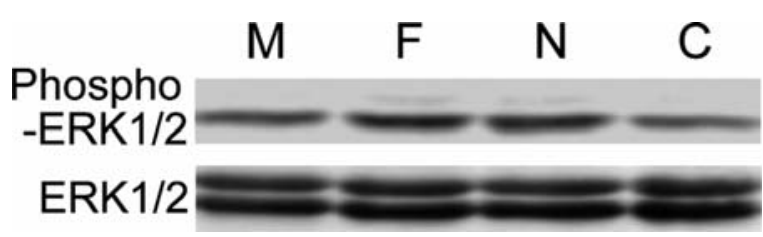

B

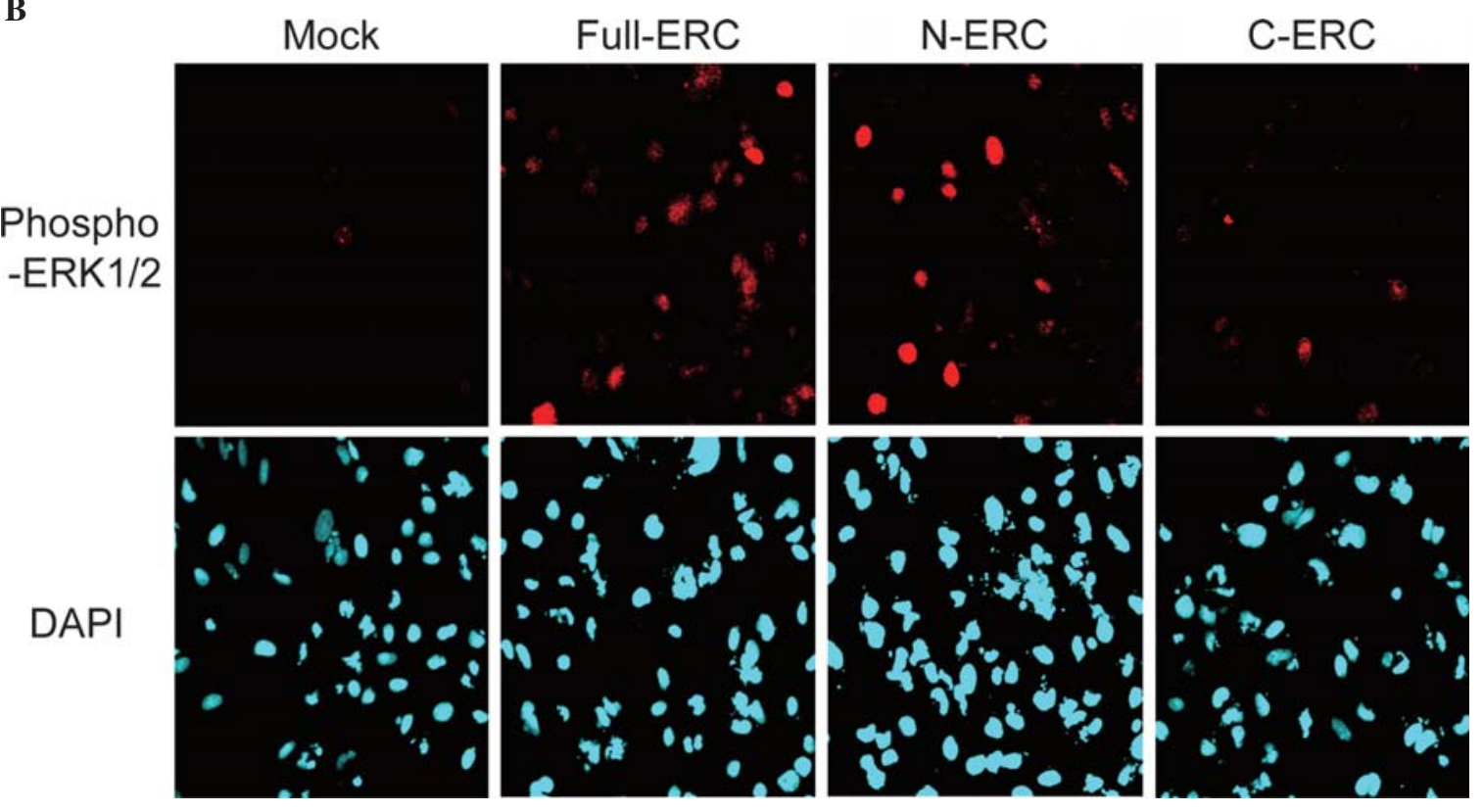

C

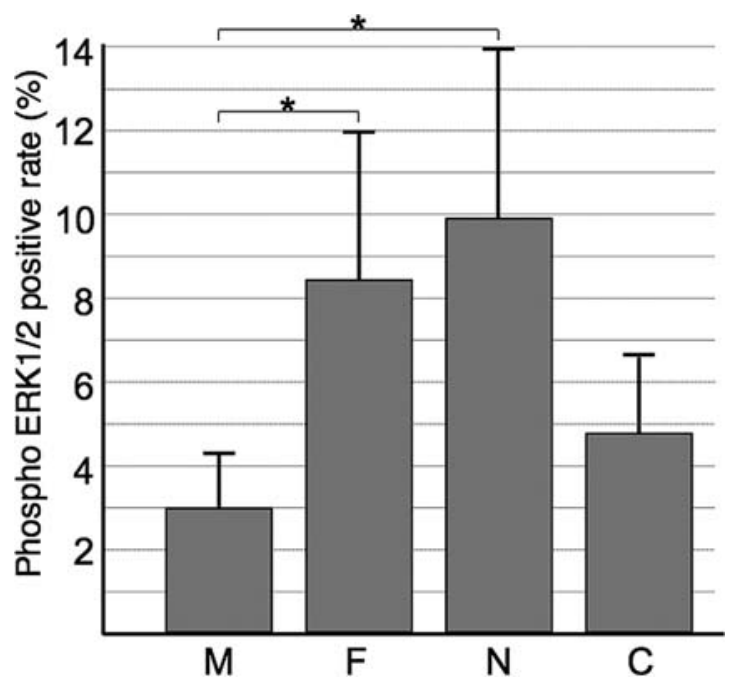

and methods. Western blotting showed that full- and N-ERC stimulated the phosphorylation of ERK1/2 (Fig. 4A). A similar result was obtained from the immunofluorescent staining analysis (Fig. 4B). The rates of phospho-ERK1/2 positive cells were significantly higher $(\mathrm{p}<0.01)$ in the full- and N-ERC transfected cell cultures than the mock or C-ERC transfected cell cultures (Fig. 4C). We also examined the effect of ERC on phosphorylation of Stat 3 and on the expression of cyclin E, but we did not detect any change in either of those markers in cells transfected with ERC expression vectors (data not shown).
Figure 4. Effect of full-, N-, and C-ERC on the phosphorylation of MAP kinase (ERK1/2, p44/42). (A) Western blotting of phospho-ERK1/2 and total ERK1/2. Twelve hours after transfection, $2 \times 10^{5}$ cells were transferred to each low adhesion surface plate ( $2 \%$ serum) and, $48 \mathrm{~h}$ later, the cells were lysed; $60 \mu \mathrm{g}$ of each lysate was run on a $10 \%$ Laemmli gel. M, F, N, and C: as in Fig. 1B. (B and C) Immunofluorescent staining of phospho-ERK1/2. Twelve hours after transfection, $1 \times 10^{5}$ cells were transferred to each glass bottom dish ( $2 \%$ serum) and, $48 \mathrm{~h}$ later, the cells were fixed with $4 \%$ paraformaldehyde for phospho-ERK1/2 staining (B). The frequency (\%) of phospho-ERK1/2-positive cells among the total population of DAPI-positive cells is shown in $(\mathrm{C}) . \mathrm{M}, \mathrm{F}, \mathrm{N}$, and $\mathrm{C}$ : same as in Fig. 1B. ${ }^{*} \mathrm{p}<0.01(\mathrm{n}=6)$.

Effect of $\mathrm{N}$-ERC secreted in culture medium on cell growth. We tried to confirm that the effect of N-ERC on the cell growth (shown in Fig. 2A) was mediated by the secreted N-ERC in medium. In anchorage-independent condition, Huh7 cells were cultured in the medium that was derived from N-ERCor mock-transfected Huh cells. The numbers of cells were counted at 24, 48 and $72 \mathrm{~h}$ after the addition of conditioned medium (Fig. 5). At $72 \mathrm{~h}$, the cells cultured in N-ERC (+) medium had significantly larger number of cells $(\mathrm{p}<0.01)$ than cells treated in mock medium. We performed similar experiment using regular culture palates that allow the 


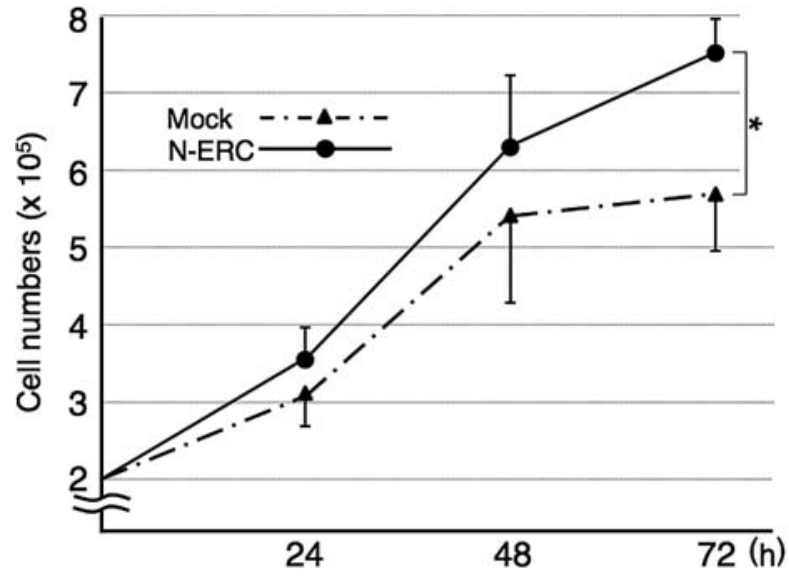

Figure 5. Effect of conditioned medium containing the secreted N-ERC on the cell growth of Huh7 in anchorage-independent condition. The conditioned medium (2\% FCS) of N-ERC- or mock-transfected Huh7 were transferred to low adhesion surface plates containing $2 \times 10^{5}$ non-treated Huh7 cells in $0.5 \mathrm{ml}$ medium. The number of cells was counted at 24,48 , and $72 \mathrm{~h}$ after the addition of conditioned medium $(\mathrm{n}=6) .{ }^{*} \mathrm{p}<0.01$.

anchorage-dependent condition. The effect of secretory N-ERC as shown in Fig. 5 was not observed in the anchoragedependent condition (data not shown).

\section{Discussion}

$\mathrm{N}$-ERC has been reported as megakaryocyte potentiating factor (MPF), a cytokine that stimulates maturation of platelets in the presence of interleukin-3 (9), but its role in tumorigenesis is unknown at present. Shiomi et al (10) and Onda et al (11) reported that the concentration of N-ERC/MPF is higher in the sera of patients with advanced MM. Similarly, its concentration is higher in the sera of Eker rats with renal cancer than in cancer-free rats, and it is secreted more abundantly by cultured cells that are more actively proliferating (12). Consideration of the published findings led us to hypothesize that secretory N-ERC promotes the proliferation of tumor cells. To test this hypothesis, we investigated whether some of full-ERC functions were also performed by secretory N-ERC in cultured cells.

Several studies have investigated the functions of full-ERC/ mesothelin using overexpression or knock-down strategies (12-14). The reported functions fall into three general categories. First, Chang et al, Rump et al and other groups $(3,13,14)$ showed that ERC/mesothelin enhances the adhesion and invasion of tumor cells. These functions are possibly mediated by C-ERC on cellular membrane. Second, Bharadwaj et al (15) showed that the protein stimulates cell proliferation by enhancing the expression of S-phase promoting cyclins/ CDKs as a result of Stat 3 activation. Third, Uehara et al and Chang et al $(16,17)$ reported that ERC/mesothelin suppresses cell death. In this study, we examined whether functions of full-ERC reported by Bharadwaj et al (15) or Uehara et al (16) may be carried out by the secretory N-ERC. We demonstrated that the secretory N-ERC suppressed cell death leading to an increase in cell number of Huh7. It enhanced phosphorylation of ERK1/2 (Fig. 4A-C), but did not affect phosphorylation of Stat3 nor expression of cyclin E (data not shown). The functions of N-ERC in our experiments were similar to those of full-ERC reported by Uehara et al (16).

In our transient expression system, the transfection efficiency was $\sim 30 \%$, evaluated by the immunofluorescent staining (data not shown). We interpreted that the effect of full- or N-ERC/mesothelin shown in Figs. 2-4 was mediated by the secreted N-ERC/mesothelin, which could influence almost $100 \%$ of cells in culture. In Fig. 5, we showed the effect of the culture medium containing the secreted N-ERC/ mesothelin. It is still possible, however, that the other cytokines induced by the overexpressed N-ERC exerted such an effect. To clarify this point, we have to purify the N-ERC and examine its effect on the cell in the future.

Uehara et al (16) described that the effect of full-ERC to increase cell number was observed only in anchorageindependent conditions. We examined the effect of N-ERC on cells both in anchorage-dependent and -independent conditions. To set up anchorage-independent conditions, we used the low adhesion surface plate (EZ-BindShut, Iwaki, Tokyo) coated with phospholipid polymers, which do not allow cells to attach on the plate surface and they grow in a form of spherocytes. The effect of medium containing N-ERC on cell number (Fig. 5) was observed only in the anchorage-independent condition. The data were compatible with the report of Uehara et al (16). However, the effect of transfected N-ERC on cell number (Figs. 2A and 3A) was observed both in the anchorage-independent and -dependent conditions (data not shown). The reason of such differences in the response of cells for the secreted, or transfected N-ERC is not clear at present.

We examined cell viability using both TUNEL to identify cell death, and BrdU incorporation to identify dividing cells. Cells expressing full- or N-ERC showed significantly reduced rates of cell death and significantly greater rates of cells with BrdU uptake. Interestingly, P-values relating to the suppression of TUNEL positivity by full- or N-ERC $(p=5.43 \mathrm{E}-10$ or $5.22 \mathrm{E}-11$ ) (Fig. 3C) were very much smaller than those found in the equivalent cultures for BrdU incorporation $(\mathrm{p}=0.0027$ or $\mathrm{p}=0.0023$ ) (Fig. $2 \mathrm{~B})$. We interpret these results as indicating that the apparent stimulation of cell growth by full- or N-ERC (Fig. 2A) resulted mainly from suppression of cell death, and, similarly, that the apparently increased rate of cells with BrdU incorporation may also result mostly from suppressed cell death.

The drawback in our study is that we have not clarified the molecular pathway of cell death suppressed by N-ERC that enhanced the phosphorylation of MAP kinase (ERK1/2). Activation of ERK1/2 is reported to inhibit cell death via suppression of Bim (BH3-only protein) that neutralizes antiapoptotic proteins such as Bcl-2 (19-21). Uehara et al showed that ERC/mesothelin enhances ERK1/2 phosphorylation, which inversely correlates with Bim levels (16). We examined the expression of Bim or Bcl-2 in Huh7 cells transfected with ERC-expression vectors, but we were not able to obtain consistent changes of their expression.

C-ERC is abundantly expressed on cell membranes in $\mathrm{MM}$, pancreatic cancer and ovarian cancer, and is known to be involved in invasion by tumor cells. Herein, we found that $\mathrm{C}$-ERC had some effect in increasing BrdU incorporation or suppressing cell death (Figs. 2B and 3A-C), although these effects were not statistically significant. Therefore, on the 
basis of our experimental results, we are unable to exclude the possibility that C-ERC also functions to stimulate cell growth.

C-ERC is currently being tested as a possible target for antibody-mediated cancer therapy. A phase I clinical study using an anti-C-ERC antibody bound to Pseudomonas exotoxin showed anti-tumor activity in patients with MM, pancreatic or ovarian cancers $(22,23)$. In contrast, although N-ERC is abundantly expressed in the sera of MM patients, its biological significance is unknown at present. We speculated that secretory $\mathrm{N}$-ERC had a cytokine-like function and could stimulate tumor growth; we tested this speculation herein, and found that it suppressed cell death and, thereby, increased total cell numbers in culture. If N-ERC can be proved to stimulate tumor progression by a cytokine-like activity in vivo, then it might be feasible to develop a new treatment strategy for MM based on the use of anti-N-ERC antibodies.

\section{Acknowledgements}

This work was supported in part by Grants-in-Aids for Scientific Research, for Cancer Research, and a consignment expense for the Molecular Imaging Program on 'Research Base for PET Diagnosis', from the Ministry of Education, Culture, Sports, Science, and Technology, and the Ministry of Health, Labor, and Welfare of Japan.

\section{References}

1. Hino O, Kobayashi E, Nishizawa M, Kubo Y, Kobayashi T, Hirayama Y, Takai S, Kikuchi Y, Tsuchiya H, Orimoto K, Kajino $\mathrm{K}$ and Takahara T: Renal carcinogenenesis in the Eker rat. J Cancer Res Clin Oncol 121: 602-605,1995.

2. Yamashita Y, Yokoyama M, Kobayashi E, Takai S and Hino O: Mapping and determination of the cDNA sequence of the Erc gene preferentially expressed in renal cell carcinoma in the Tsc2 gene mutant (Eker) rat model. Biochem Biophys Res Commun 275: 134-140, 2000.

3. Chang K and Pastan I: Molecular cloning of mesothelin, a differentiation antigen present on mesothelium, mesotheliomas, and ovarian cancers. Proc Natl Acad Sci USA 93: 136-140, 1996.

4. Chang K, Pastan I and Willingham MC: Frequent expression of the tumor antigen CAK1 in squamous-cell carcinomas. Int J Cancer 51: 548-554, 1992.

5. Frierson HF Jr, Moskaluk CA, Powell SM, Zhang H, Cerilli LA, Stoler MH, Cathro H and Hampton GM: Large-scale molecular and tissue microarray analysis of mesothelin expression in common human carcinomas. Hum Pathol 34: 605-609, 2003.

6. Cao D, Ji H and Ronnett BM: Expression of mesothelin, fascin, and prostate stem cell antigen in primary ovarian mucinous tumors and their utility in differentiating primary ovarian mucinous tumors from metastatic pancreatic mucinous carcinomas in the ovary. Int J Gynecol Pathol 24: 67-72, 2005.

7. Hough CD, Sherman-Baust CA, Pizer ES, Montz FJ, Im DD, Rosenshein NB, Cho KR, Riggins GJ and Morin PJ: Large-scale serial analysis of gene expression reveals genes differentially expressed in ovarian cancer. Cancer Res 60: 6281-6287, 2000.
8. Argani P, Iacobuzio-Donahue C, Ryu B, Rosty C, Goggins M, Wilentz RE, Murugesan SR, Leach SD, Jaffee E, Yeo CJ, Cameron JL, Kern SE and Hruban RH: Mesothelin is overexpressed in the vast majority of ductal adenocarcinomas of the pancreas: identification of a new pancreatic cancer marker by serial analysis of gene expression (SAGE). Clin Cancer Res 7: 3862-3868, 2001.

9. Kojima T, Oh-Eda M, Hattori K, Taniguchi Y, Tamura M, Ochi N and Yamaguchi N: Molecular cloning and expression of megakaryocyte potentiating factor cDNA. J Biol Chem 270: 21984-21990, 1995.

10. Shiomi K, Miyamoto H, Segawa T, Hagiwara Y, Ota A, Maeda M, Takahashi K, Masuda K, Sakao Y and Hino O: Novel ELISA system for detection of N-ERC/mesothelin in the sera of mesothelioma patients. Cancer Sci 97: 928-932, 2006.

11. Onda M, Nagata S, Ho M, Bera TK, Hassan R, Alexander RH and Pastan I: Megakaryocyte potentiation factor cleaved from mesothelin precursor is a useful tumor marker in the serum of patients with mesothelioma. Clin Cancer Res 12: 4225-4231, 2006.

12. Nakaishi M, Kajino K, Ikesue M, Hagiwara Y, Kuwahara M, Mitani H, Horikoshi-Sakuraba Y, Segawa T, Kon S, Maeda M, Wang T, Abe M, Yokoyama M and Hino O: Establishment of the enzyme-linked immunosorbent assay system to detect the amino terminal secretory form of rat Erc/Mesothelin. Cancer Sci 98: 659-664, 2007.

13. Rump A, Morikawa Y, Tanaka M, Minami S, Umesaki N, Takeuchi M and Miyajima A: Binding of ovarian cancer antigen CA125/MUC16 to mesothelin mediates cell adhesion. J Biol Chem 279: 9190-9198, 2004.

14. Gubbels JA, Belisle J, Onda M, Rancourt C, Migneault M, Ho M, Bera TK, Connor J, Sathyanarayana BK, Lee B, Pastan I and Patankar MS: Mesothelin-MUC16 binding is a high affinity, $\mathrm{N}$-glycan dependent interaction that facilitate peritoneal metastasis of ovarian tumors. Mol Cancer 5: 50, 2006.

15. Bharadwaj U, Li M, Chen C and Yao Q: Mesothelin-induced pancreatic cancer cell proliferation involves alteration of cyclin $\mathrm{E}$ via activation of signal transducer and activator of transcription protein 3. Mol Cancer Res 6: 1755-1765, 2008.

16. Uehara N, Matsuoka Y and Tsubura A: Mesothelin promotes anchorage-independent growth and prevents anoikis via extracellular signal-regulated kinase signaling pathway in human breast cancer cells. Mol Cancer Res 6: 186-193, 2008.

17. Chang MC, Chen CA, Hsieh CY, Lee CN, Su YN, Hu YH and Cheng WF: Mesothelin inhibits paclitaxel-induced apoptosis through the PI3K pathway. Biochem J 424: 449-458, 2009.

18. Ishikawa K, Segawa T, Hagiwara Y, Maeda M, Abe M and Hino O: Establishment of novel $\mathrm{mAb}$ to human ERC/mesothelin useful for study and diagnosis of ERC/mesothelin-expressing cancers. Pathol Int 59: 161-166, 2009.

19. Fukazawa H, Noguchi K, Masumi A, Murakami Y and Uehara Y: BimEL is an important determinant for induction of anoikis sensitivity by mitogen-activated protein/extracellular signalregulated kinase kinase inhibitors. Mol Cancer Ther 3: 1281-1288, 2004.

20. Reginato MJ, Mills KR, Paulus JK, Lynch DK, Sgroi DC, Debnath J, Muthuswamy SK and Brugge JS: Integrins and EGFR coordinately regulate the pro-apoptotic protein Bim to prevent anoikis. Nat Cell Biol 5: 733-740, 2003.

21. Yang JM, O'Neill P, Jin W, Foty R, Medina DJ, Xu Z, Lomas M, Arndt GM, Tang Y, Nakada M, Yan L and Hait WN: Extracellular matrix metalloproteinase inducer (CD147) confers resistance of breast cancer cells to anoikis through inhibition of Bim. J Biol Chem 281: 9719-9727, 2006.

22. Hassan R, Bera T and Pastan I: Mesothelin: A new target for immunotherapy. Clin Cancer Res 10: 3937-3942, 2004.

23. Hassan R and Ho M: Mesothelin targeted cancer immunotherapy. Eur J Cancer 44: 46-53, 2008. 